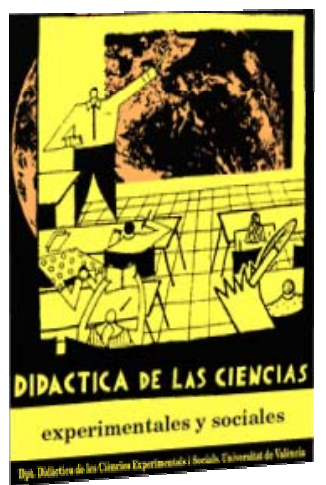

\title{
Vivir la Historia: Posibilidades de la empatía histórica para motivar al alumnado y lograr una comprensión efectiva de los hechos históricos
}

\author{
Living History: Possibilities of historical \\ empathy to motivate students and achieve an \\ effective understanding of historical events
}

DOI: 10.7203/DCES.36.12993

\author{
Olga Doñate Campos \\ Universitat Jaume I, al362425@uji.es \\ Carmen Ferrete Sarria \\ Universitat Jaume I, ferrete@fis.uji.es
}

\begin{abstract}
RESUMEN: Este trabajo investiga las posibilidades de la empatía histórica para motivar al alumnado y lograr un aprendizaje basado en una comprensión profunda de la Historia, evitando el aprendizaje mnemotécnico y superficial. Para ello se implementan actividades de empatía histórica en aulas de secundaria, analizando su aplicación mediante la definición de unos niveles de comprensión del contexto histórico y a partir de la recolección de datos sobre la capacidad de motivación que presentan estas actividades.
\end{abstract}

Palabras Clave: empatía histórica, aprendizaje significativo, motivación, aprendizaje activo, educación secundaria.

ABSTRACT: This work investigates the possibilities of historical empathy to motivate students and achieve a learning based on a deep understanding of history, avoiding mnemonic and superficial learning. To this end, historical empathy activities were implemented in secondary classrooms. Their application is analyzed by defining levels of understanding of the historical context and by collecting data on the motivational capacity of these activities.

KEYWORDS: historical empathy, significant learning, motivation, active learning, secondary school.

Fecha de recepción: agosto de 2018

Fecha de aceptación: marzo de 2019

Este trabajo ha sido tutorizado por Carmen Ferrete Sarria, Licenciada en Filosofía por la Universidad de Valencia y Doctora en Filosofía por la Universitat Jaume I; Máster en Bioética; Profesora asociada desde el año 2005 del Área de Filosofía Moral de la Universitat Jaume I de Castellón; Profesora de Secundaria, en la actualidad en el IES Álvaro Falomir (Almassora). 


\section{INTRODUCCIÓN Y JUSTIFICACIÓN DEL TÍTULO DE ESTUDIO}

La Historia se percibe por parte del alumnado como una materia aburrida y lejana, desconectada de la realidad (Pagés y Santisteban, 2013). La metodología utilizada por los docentes en las clases de Historia, a pesar de la corriente de renovación que se está produciendo en la enseñanza, es muy parecida a la del pasado (Pericacho, 2015). Predomina una enseñanza que utiliza metodologías tradicionales, fundamentadas básicamente en la memorización mecánica en detrimento de la creatividad, el aprendizaje comprensivo y la reflexión. Las clases que siguen una metodología tradicional no promueven la formación del alumnado en las competencias que pide la sociedad actual, como lo son la capacidad para seleccionar y procesar la información, para gestionar los problemas de nuestro mundo o para integrarse en el ámbito laboral (Pericacho, 2015). La repetición mecánica de fechas y datos históricos no desarrolla ninguna otra habilidad que no sea la de ejercitar la memoria, habilidad cada vez más devaluada en una sociedad donde solo es necesario llevarse la mano al bolsillo y sacar el smartphone para buscar en Google aquello que deseamos saber. Nos encontramos frente a "una realidad multicultural, globalizada y tecnológica, que reclama discernir cuáles son los conocimientos y habilidades que realmente deben conocer los alumnos (Canals, 2008: 332). Por lo tanto, cada vez son más las voces que se alzan para reclamar avances en la sociedad que equiparen escuela y sociedad. La educación debe adaptarse al cambio social, formando alumnos competentes, capaces de moverse en el mundo actual.

Entre las diversas metodologías existentes que pueden ayudar al alumnado a evitar un aprendizaje superficial y mnemotécnico de la Historia, en este trabajo se propone la empatía histórica. Los objetivos del presente trabajo son investigar si la empatía histórica es un medio válido para mejorar la comprensión del alumnado sobre los hechos históricos, logrando así un enfoque basado en el razonamiento y el sentido crítico; así como indagar en la capacidad del aprendizaje empático para conseguir una mayor motivación en el alumnado hacia la actividad escolar.

Con tal fin, hemos llevado a cabo el diseño y la aplicación de dos actividades de empatía histórica, buscando responder a los interrogantes suscitados sobre las posibilidades de la empatía histórica como elemento transformador del aprendizaje. Para ello hemos definido una serie de niveles de comprensión del contexto histórico y hemos comprobado la mayor o menos adecuación del alumnado a los mismos.Asimismo hemos observado y recolectado información sobre la capacidad motivadora de estas actividades. La implementación de las actividades de empatia histórica se ha llevado a cabo en un curso de $4^{\circ}$ de la ESO, en un centro de Educación Secundaria, durante el prácticum del Máster Universitario en Profesor/a de Educación Secundaria Obligatoria y Bachillerato, Formación Profesional y Aprendizaje de Idiomas de la Universidad Jaime I. Por lo tanto, el presente trabajo se trata de una adaptación de un proyecto más extenso, un trabajo final de máster homónimo: Viure la Història un aprenentatge empàtic.

\section{MARCO TEÓRICO}

\section{1. ¿Qué es la empatía histórica?}

En primer lugar, resulta necesario definir qué entendemos por empatía histórica. Lazarouk (2008) señala que los historiadores dan un significado al concepto de empatía diferente al que le otorgan los psicólogos y psiquiatras, puesto que emplean la empatía como una herramienta para la investigación histórica. Yilmaz recoge la siguiente definición:

Downey, que prefiere el término "toma de perspectiva" al concepto “empatía histórica”, define el término como la habilidad de comprender los marcos de referencia de los personajes históricos, en base a hechos y acontecimientos históricos, sin tratar de identificarse o simpatizar con sus sentimientos. (Yilmaz, 2007: 332) 
Según esta definición se busca un estudio del pasado racional, no emotivo. Es decir, para realizar un ejercicio de empatía histórica con éxito no es necesaria ninguna implicación emocional del agente que lo realiza. Simplemente se busca la comprensión de las mentalidades, del comportamiento de los personajes históricos, no una identificación afectiva con estos. El mismo término "toma de perspectiva" sugiere un alejamiento emocional respecto a los personajes históricos estudiados.

Endacott y Brooks (2013) en cambio, puntualizan que un acercamiento afectivo mejora la capacidad de comprensión y contextualización de la Historia. Tratar el ámbito afectivo de la empatía histórica no implica una práctica deficiente de la vertiente cognitiva. Ambos aspectos son complementarios. "El proceso de empatía histórica es tanto afectivo como cognitivo. Es cognitivo porque requiere razonar sobre el modo en que las evidencias encajan entre sí. Es afectivo porque trata de imaginar qué podría haber sentido un personaje histórico determinado" (Davison, 2012b:12-13). Resulta complicado ejercer la empatía histórica prescindiendo de toda implicación emocional, puesto que precisamente la observación de nuestros propios sentimientos y la identificación de los mismos en otras personas es aquello que nos capacita para comprenderlos. Lo cual no significa que esta aproximación, tal y como ha señalado Davison (2012b), nos pueda desproveer de nuestro razonamiento crítico. Podemos comprender a un determinado personaje histórico utilizando nuestro registro personal de sentimientos pero esto no implica que no seamos capaces de analizar de un modo racional a dicho personaje. Por ejemplo, es posible comprender el antisemitismo desde el punto de vista emocional (ser capaz de percibir cómo la población de la Alemania nazi creía realmente que los judíos eran una amenaza, el odio que sentían hacia quienes consideraban seres viles y malintencionados...) pero esto no implica compartirlo.

Una vez tratada la definición de empatía histórica, resulta conveniente definir las características, dimensiones o elementos (la terminología depende del autor) que la configuran. Podemos observar que cada autor, se decanta por una serie de elementos. Foster (1999) identifica seis cualidades de la empatía histórica y Endacott (2010) recoge tres elementos. Expondremos aquí el modelo de las dimensiones de Davison (2012a p.123), puesto que es el que posteriormente utilizaremos en el análisis de los resultados de las actividades implementadas. Distingue entre:

Dimensiones afectivas:

- Mentalidad abierta: es la disposición por entender otros puntos de vista.

- Compasión: aglutina tanto ser compasivo como sensible y tolerante hacia otras personas.

- Imaginación: ayuda a los estudiantes a figurarse cómo era el pasado. Dimensiones cognitivas:

- Búsqueda de evidencias: incluye contrastar diversas teorías sobre el pasado, utilizar un amplio espectro de pruebas y analizar la fiabilidad y utilidad de las evidencias históricas.

- Buscar múltiples perspectivas: para interpretar el pasado de acuerdo al análisis de diversos puntos de vista.

Evitar el presentismo: a fin de interpretar las creencias y comportamientos de los personajes históricos del mejor modo posible.

\section{2 ¿Cómo puede beneficiar a los estudiantes la empatía histórica?}

La empatía histórica presenta una serie de ventajas que convierten su uso en el aula en un recurso imprescindible para cualquier docente que imparta esta disciplina. La empatía histórica no solo puede ser utilizada como un modo de dinamizar y mejorar el aprendizaje de la Historia, sino que sus implicaciones abarcan también el desarrollo cívico y emocional del alumnado.

\section{¿Fomenta la motivación?}

Debido a la estrecha relación existente entre emoción y motivación (Beltrán et al., 1987), podemos suponer que implicando a los alumnos emocionalmente en el aprendizaje de la Historia 
despertaremos su interés. Asegurando el debido rigor histórico, se trata de huir de un análisis frío e impersonal de un determinado contexto histórico, que provoque indiferencia en los alumnos y se perciba simplemente como un tema más en el manual de texto. Kohlmeier, después de haber llevado a cabo ejercicios de empatía histórica en el aula, señala que:

\begin{abstract}
Mis estudiantes estuvieron motivados para leer documentos históricos complejos y confusos y buscar información, así como estudiar el contexto histórico porque sintieron afecto por la mujer a la que estudiaron. Notablemente, tanto los chicos como las chicas, evidenciaron esta conexión. Observé estudiantes de catorce años expresar ideas con pasión y preocupación sobre las vidas de mujeres pertenecientes a periodos históricos lejanos al suyo. Sus comentarios en los seminarios eran siempre una indicación de su comprensión y de las conexiones emocionales establecidas con las experiencias de los autores. Sin esta conexión, no estoy segura que mis alumnos hubieran realizado la ardua tarea que les encomendé (Kohlmeier, 2006: 52).
\end{abstract}

Guillén (2016) a su vez, señala que las actividades de empatía histórica resultan motivadoras porque evitan la repetición de contenidos y el aprendizaje pasivo, implicando al estudiante en las vivencias y cosmovisiones del pasado. Por lo tanto, las actividades que emplean la empatía histórica en el aula suponen una forma de romper la rutina y de evitar las lecciones magistrales, para invitar al alumnado a reflexionar y participar activamente. Asimismo, el aprendizaje mediante la empatía histórica es necesariamente activo, puesto que la participación del estudiante es imprescindible para lograr que este se desarrolle adecuadamente. Por ejemplo, la visualización de una película es un recurso empleado o recomendado por varios profesores que tratan en el aula la empatía histórica (Davison, 2012b; Guillén, 2016; Cruz y Pizarro, 2014) pero siempre como un elemento más para favorecer el proceso de empatía histórica, el cual incluye necesariamente una producción o reflexión personal del alumnado.

Tal como Sierra (2013) señala, un gran número de psicólogos y especialistas en educación defienden la importancia del aprendizaje activo en el proceso didáctico. Este mejora la atención de los estudiantes, puesto que resulta probable que no sean capaces de mantener la atención ante una lección expositiva. El alumnado consigue una comprensión más profunda de los conceptos, se beneficia de la interacción con los compañeros al aula y mejora su actitud hacia el aprendizaje.

Considerando que la motivación es un efecto de las actividades de empatía histórica que suelen mencionar todos los autores que las llevan a cabo en el aula (Davison, 2012a; Kohlmeier, 2006; Guillén, 2016; Downey, 1995; Cruz y Pizarro, 2014), podemos concluir que a pesar de la carencia de estudios en el campo de la pedagogía y la psicología que interrelacionen motivación y empatía histórica, estas se encuentran intrínsecamente relacionadas. Por tanto, cualquier docente debería considerar la necesidad de incorporar actividades de empatía histórica en el aula como un modo, si no de vertebrar la actividad docente de la materia, al menos de enriquecerla y completarla.

\title{
¿Mejora la capacidad de comprender y razonar?
}

Kohlmeier (2006) menciona que mediante los ejercicios de empatía histórica sus estudiantes se dieron cuenta de la complejidad y diversidad que puede ofrecer esta materia. Descubrieron que la Historia no se basa en la simple memorización de hechos, sino que aprendieron a "leer textos críticamente, explorar múltiples perspectivas de un hecho histórico, considerar varias interpretaciones históricas y sus implicaciones, y defender argumentos a partir de evidencias históricas” (Kohlmeier, 2006: 54). Mootz (2014) por su parte, propugna que las actividades de empatía histórica que permiten al alumnado tratar fuentes de primera mano, compartir opiniones mediante el debate y realizar una tarea de análisis, derivan en una mejora de la habilidad de los adolescentes para razonar en el campo de la Historia. Asimismo, Davison (2012a) realiza una investigación con su alumnado a fin de probar esta premisa. Una clase recibe, en primer lugar, lecciones centradas en el aspecto afectivo mediante la empatía histórica, y otra clase centradas en el 
ámbito cognitivo. Ambas clases reciben los dos tipos de lecciones, tan solo varía el orden. A lo largo de todo este proceso, los estudiantes realizan una serie de tareas que miden su capacidad para comprender la Historia. Después de una tarea inicial que muestra que ambas clases tienen niveles similares en su habilidad de comprensión histórica, Davison presenta una segunda tarea donde cada una de las clases tan solo habrá recibido lecciones enfocadas a la vertiente cognitiva o afectiva. Los resultados muestran que los estudiantes centrados en la afectividad, presentan mejores resultados que aquellos cuyas lecciones se orientan al aspecto cognitivo. El hecho de indagar en los motivos que trajeron a un determinado personaje histórico a comportarse de una cierta forma suponen, en el individuo, desarrollar su capacidad para comprender las motivaciones ajenas y la complejidad del proceso de toma de decisiones. "La empatía histórica coloca a los alumnos en una posición ventajosa para analizar y evaluar sus propias creencias y acciones, así como las de los otros con los que ellos se relacionan en el tiempo presente” (Endacott y Brooks, 2013: 45). Asimismo, Davison no solo analiza como la afectividad puede mejorar el análisis histórico, sino que también indaga en su capacidad motivadora. Los resultados muestran que los estudiantes que primero han tratado los materiales relacionados con la afectividad, muestran mayor interés y entusiasmo hacia la tarea (Davison, 2012a).

\section{FASE DE APLICACIÓN}

\subsection{Objetivos y contextualización de las actividades}

\section{Objetivos}

En la introducción hemos esbozado los objetivos generales que buscamos alcanzar mediante este trabajo. Cabe señalar asimismo los objetivos específicos que buscamos obtener a partir de la implementación de las actividades de empatía histórica:

- Discernir si las actividades propuestas permiten al alumnado lograr una comprensión profunda y significativa de los hechos históricos.

- Observar, en función de la participación de los alumnos la capacidad de las actividades realizadas para motivarles.

- Establecer el nivel de satisfacción e interés del alumnado respecto a las actividades propuestas y ejecutadas.

\section{Contextualización}

Este trabajo se ha llevado a cabo con dos grupos de alumnos $4^{\circ}$ de la ESO, $4^{\circ} \mathrm{A}$ y $4^{\circ} \mathrm{B}$, de 27 y 26 alumnos respectivaemnte, que cursan la materia Geografía e Historia en el Centro diocesano Mater Dei, ubicado en Castellón, Castellón de la Plana.

Como se ha indicado en la introducción, este trabajo se desarrolla durante el prácticum del Máster Universitario en Profesor/a de Educación Secundaria Obligatoria y Bachillerato, Formación Profesional y Aprendizaje de Idiomas de la Universidad Jaime I. Por lo tanto, la implantación del mismo se ha limitado espacialmente al centro en el cual se ha ejercido el prácticum y se ha adaptado cronológicamente al marco de las prácticas docentes (la impartición de una unidad didáctica por el alumno en prácticas: de 8 a 10 sesiones lectivas de 55 minutos de duración).

\section{Implementación de las actividades}

Se han propuesto dos actividades. La actividad A:“¿Cómo explicamos el auge de los fascismos?” y la actividad B“: Totalitarismo en primera persona”. Se han llevado a cabo a lo largo de diez sesiones lectivas, cinco en cada grupo de alumnos. La primera sesión se ha destinado a la 
actividad A, la segunda, tercera sesión y cuarta sesión a la actividad B. Finalmente la quinta sesión se ha dedicado a responder dudas de los alumnos y a realizarles preguntas, tanto escritas como orales, a fin evaluar la efectividad de las actividades planteadas en términos de motivación y comprensión de la Historia. Ambas actividades son precedidas, en sesiones anteriores, de la explicación del contenido pertinente: "El fascismo italiano y el nazismo alemán: orígenes y desarrollo", que se inserat en el bloque temático "Las crisis de Entreguerras y la II Guerra Mundial” de la asignatura Geografía e Historia, regulada por el decreto 87/2015 de 5 de junio, que establece el currículum y la ordenación general de la Educación Secundaria Obligatoria y el Bachillerato en la Comunidad Valenciana.

Antes de exponer la metodología que hemos seguido en las actividades es necesario explicar en qué consiste cada una de ellas.

\section{Actividad A: ¿Cómo explicamos el auge de los fascismos?}

El tema que se ha tratado, el ascenso de Hitler y Mussolini al poder, son hechos que habitualmente resultan abstractos para la población actual. Parece inconcebible la existencia, en un pasado tan reciente, de dictadores que hicieran tan explícito su ideario y que, a pesar de esto, consiguieran apoyo popular. Los interrogantes sobre cómo fue posible que el Holocausto ocurriera, cómo estos personajes históricos consiguieron el poder... acaban por abordar a cada individuo al menos una vez en su vida. Resulta sumamente complicado comprender verdaderamente este periodo histórico, especialmente si nos limitamos a la habitual y breve enumeración de las causas que aparecen en los libros de texto. Los estudiantes leen estas causas y las pueden tratar de comprender (con éxito o no) o simplemente memorizarlas para aprobar el examen, pero no empatizarán realmente con los acontecimientos mencionados si no profundizan en el tema. No es lo mismo saber que los alemanes estaban frustrados por las duras reparaciones de guerra exigidas en Alemania que leer el testimonio de un alemán relatando el hambre, el frío y la desesperación sufridas en la posguerra, así como el profundo sentimiento de injusticia que experimentó.

La primera actividad (sesión 1) planteada de este proyecto supone realizar una comparativa entre uno o varios hechos históricos y sucesos actuales. En este caso, se busca que los estudiantes comparen el ascenso del fascismo italiano y alemán con el contexto actual de ascenso de la extrema derecha, concretamente, el auge de Donald Trump y Marine Le Pen. Se busca que los estudiantes encuentren las causas comunes del apoyo popular que ha recibido la derecha radical en periodos diferentes de la Historia. Endacott y Brooks (2013: 53) señalan que:

Uno de los objetivos de la empatía histórica es comprender que tanto las ideas del pasado como del presente son el resultado del contexto histórico. Las actividades de reflexión pueden orientar a los estudiantes hacia la investigación de situaciones en el pasado similares a las del presente y que por lo tanto, puedan explicar circunstancias y hechos actuales.

Hitler y Mussolini canalizan el descontento de la población, un sentimiento de decepción que actualmente es cada vez más patente, y que como antes, está siendo instrumentalizado por varias fuerzas políticas. Entender el porqué de una determinada conducta (el apoyo de la población a mensajes políticos radicales), resulta más sencillo si vemos esta misma conducta en nuestra realidad actual. Por ejemplo, resulta sumamente complicado para los estudiantes comprender los motivos que hay detrás de las acciones arriesgadas de algunos héroes de guerra para obtener reconocimiento, por más que se les intente explicar la mentalidad de la época. Pero si hacemos un paralelismo actual, utilizando como ejemplo los casos de los jóvenes que incluso arriesgan su vida para conseguir un video de youtube impactante que les dé seguidores, resulta más tangible un concepto abstracto. 
La segunda actividad propuesta consiste en la visualización de la película La Ola (sesiones 2 y 3) que trata la facilidad con la cual los seres humanos se adscriben a una autocracia, y la posterior redacción, por parte de cada alumno, de una carta donde este explique como cree que reaccionaría ante una situación como la que se muestra al film. A su vez, los estudiantes de la otra línea de $4^{\circ}$, deben revisar las cartas de los compañeros e indicar si están o no de acuerdo con el contenido de estas. Es decir, si consideran que realmente el autor de la carta reaccionaría tal como él o ella ha asegurado.

En general, las películas que tratan acontecimientos históricos son una herramienta adecuada para practicar la empatía histórica, puesto que permiten la vinculación afectiva del alumnado con los personajes históricos tratados (Davison, 2012b). En primer lugar, la visualización de la película ya supone un importante ejercicio de empatía histórica, puesto que "La Ola” profundiza en la psicología y los motivos de varios adolescentes para rechazar o adoptar un movimiento de características fascistas. Es un relato cercano, que aproxima una situación abstracta a la realidad más inmediata de los estudiantes.

En la segunda parte de la actividad (sesión 4) los alumnos llevarán a cabo la redacción de una carta expresando como creen que actuarían en una situación igual o similar. Esta es una forma, a través del juego de roles, de empatizar con aquellos que dieron su apoyo a Hitler y Mussolini. Supone imaginarse en la situación descrita, ponerse totalmente en la piel de estas personas. Así, los estudiantes pueden asimilar que una dictadura es posible y reconocer los factores que influyen en el desarrollo esta. Aunque los estudiantes no sean capaces de pensar como actuarían realmente, puesto que esto requiere un ejercicio de introspección de un elevado grado de dificultad, las cartas les pueden ayudar a empatizar históricamente, puesto que facilitan que el alumno sea consciente de los factores que podrían influir al resto de personas.

\subsection{Metodología}

\section{Diseño de las actividades}

Para diseñar y desarrollar las actividades de empatía histórica nos hemos basado para en las pautas ofrecidas por los autores Kohlmeier (2006) y Pagès (2007), que se presentan un modelo de implementación que se subdivide en 3 fases coincidentes en ambos autores.

TABLA 1. Pautas de implementación de actividades de empatía histórica

\begin{tabular}{|c|c|c|}
\hline $\begin{array}{l}\text { Primera fase: Presentación de la } \\
\text { actividad }\end{array}$ & Actividad A & Actividad B \\
\hline $\begin{array}{l}\text {-Kohlmeier aconseja, tal como ella } \\
\text { misma realiza con su alumnado, presentar } \\
\text { un misterio para excitar su curiosidad. O } \\
\text { bien, una idea que genere controversia y } \\
\text { sea debatida. } \\
\text {-Pagès señala que el docente debe iniciar } \\
\text { las actividades de empatía histórica } \\
\text { planteando el conocimiento histórico de } \\
\text { forma problemática.Se trata de presentar } \\
\text { una dificultad que los estudiantes quieran } \\
\text { resolver y que por lo tanto, los motive } \\
\text { para indagar y buscar información. }\end{array}$ & $\begin{array}{l}\text { Hemos utilizado la misma } \\
\text { pregunta que da inicio a la } \\
\text { película para comenzar nuestra } \\
\text { actividad: } \\
\\
\text {-“¿Sería posible que se } \\
\text { volviera a producir una } \\
\text { dictadura en Alemania?” a fin } \\
\text { de suscitar un pequeño debate } \\
\text { antes del inicio de la actividad } \\
\text { que despierte su curiosidad. }\end{array}$ & $\begin{array}{l}\text {-“¿Votaríais a Hitler?” a fin de } \\
\text { provocar, al igual en el la } \\
\text { actividad A, un debate entre los } \\
\text { alumnos. }\end{array}$ \\
\hline
\end{tabular}

Fuente: adaptado de Kohlmeier (2006) y Pagès (2007) 
TABLA 2. Pautas de implementación de actividades de empatía histórica

\section{Segunda fase: Obtención de Actividad A información}

-Kohlemeier propone la Para llevar a cabo esta actividad, ha sido lectura de fuentes primarias empleado material audiovisual: por parte de los estudiantes, puesto que estas muestran -Fragmentos de documentales y videos perfectamente, a través del donde votantes de Donald Trump y Le estilo de escritura y del Pen exponen sus razones para dar su pue $\begin{array}{ll}\text { contenido, cómo la gente se } & \text { apoyo a estos candidatos (BBC Mundo, } \\ \text { comunicaba y pensaba. } & \text { 2017; Évole y Lara, 2017; }\end{array}$

$$
\text { 2017; Évole y Lara, }
$$

-Pagès señala que el profesor tiene que dar oportunidades al -Fragmentos de debates y discursos de estudiante para analizar las estos políticos contemporáneos (Évole y fuentes $\mathrm{y}$ las evidencias existentes.

\section{Actividad B}

Si bien la película no es ninguna fuente histórica, complementada con el contenido teórico correspondiente supone una herramienta de suma utilidad puesto que muestra cómo las personas pueden adscribirse a una autocracia. Se intenta así que los estudiantes puedan de ponerse en el lugar de las personas que dieron su apoyo a Lara, 2017; HispanTV, 2017; LA que se considera de gran valor SEXTA, 2014) y de Hitler y Mussolini para prevenir una situación (MusicaPatriota, 2015; Profesora de parecida en el futuro, tal como Geografía e Historia, 2015). Pagès (2008) y Endacott $y$ Brooks (2013) señalan.

-Textos donde los contemporáneos de Hitler y Mussolini cuentan de primera mano la percepción que tenían de ellos (Neitzel y Welzer, 2012; René, 2010).

Fuente: adaptado de Kohlmeier (2006) y Pagès (2007)

TABLA 3. Pautas de implementación de actividades de empatía histórica

Tercera fase: Debate

- Kohlmeier propone que en el Los alumnos han respondido a Se les ha ofrecido una serie de momento en que los estudiantes han diversas preguntas que preguntas durante la adquirido una base teórica deben buscaban tanto conectarlos visualización de la película, compartir y rebatir ideas mediante el afectivamente con el tema antes de la elaboración de la debate. De este modo los estudiantes tratado, como fomentar el carta para orientarles en la pueden comprender en mayor medida debate. Ejemplos:

los conceptos complejos, puesto que durante el debate estos se analizan desde varios puntos de vista, se clarifican... Además, la autora señala que ella lanza preguntas a los estudiantes para incitar -“Hemos visto como Mussolini -“¿Crees que te opondrías a tus el debate en las cuales estos necesitan y Hitler empleaban un amigos si todos, excepto tú, tomar el punto de vista del personaje nacionalismo exaltado en sus estuviesen a favor de la Ola?”, $\begin{array}{lll}\text { histórico estudiado para responder. } & \text { discursos. ¿Por qué pensáis -“¿Qué opinión tienes de los }\end{array}$ que este tipo de mensaje personajes, te sientes -Pagès defiende la necesidad de exponer funcionó? ¿¿Pensáis que identificado/a con ellos?” oralmente las conclusiones a las que han actualmente todavía se llegado los alumnos y debatirlas con el utiliza?”. resto. redacción de la misma y tras finalizar y compartir las cartas para estimular el debate. Ejemplos: 


\section{Niveles de análisis de los resultados}

A fin de analizar los resultados, nos hemos basado en una metodología cualitativa fundamentada principalmente en la observación. Durante las sesiones 1-4 se ha utilizado una rúbrica y durante la quinta sesión los estudiantes han respondido a un cuestionario.

TABLA 4. Pautas de análisis de actividades de empatía histórica

\begin{tabular}{|c|c|c|c|c|}
\hline \multirow{3}{*}{ 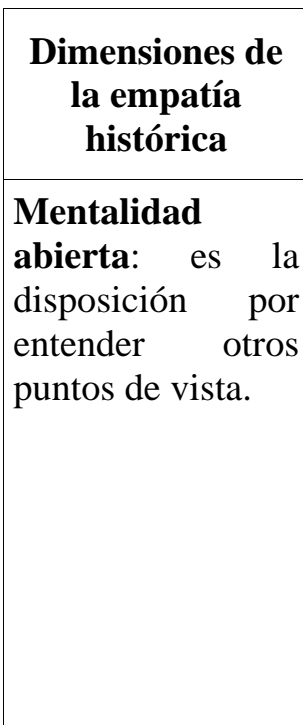 } & \multicolumn{4}{|c|}{ Niveles } \\
\hline & $\begin{array}{l}\text { 1- Requiere } \\
\text { mejoras }\end{array}$ & 2-Bueno & 3- Satisfactorio & 4-Excelente \\
\hline & $\begin{array}{l}\text { El alumno no } \\
\text { comprende un } \\
\text { punto de vista que } \\
\text { no sea el propio. }\end{array}$ & $\begin{array}{lr}\text { El alumno } \\
\text { comprende r las } \\
\text { motivaciones } \\
\text { intereses ajenos. }\end{array}$ & $\begin{array}{l}\text { El alumno } \\
\text { comprende } \\
\text { motivaciones e } \\
\text { intereses ajenos } \\
\text { asumiendo que } \\
\text { estos rastán } \\
\text { condicionados y } \\
\text { son consecuencia } \\
\text { de } \\
\text { determinado un } \\
\text { contexto r } \\
\text { histórico. }\end{array}$ & $\begin{array}{l}\text { El alumno } r \text { comprende } \\
\text { motivaciones e } \\
\text { ajenos asumiendo que estos } \\
\text { están condicionados y son } \\
\text { consecuencia de un } \\
\text { determinado r contexto } \\
\text { histórico y es capaz de } \\
\text { deducir estas motivaciones } \\
\text { e intereses si posee } \\
\text { información suficiente } \\
\text { sobre el contexto. }\end{array}$ \\
\hline $\begin{array}{l}\text { Buscar múltiples } \\
\text { perspectivas: } \\
\text { para interpretar el } \\
\text { pasado de acuerdo } \\
\text { al análisis de } \\
\text { diversos puntos de } \\
\text { vista. }\end{array}$ & $\begin{array}{lr}\text { El alumno } & \text { no } \\
\text { discierne } & \text { la } \\
\text { multiplicidad } & \text { de } \\
\text { factores } & \text { que } \\
\text { pueden explicar un } \\
\text { hecho histórico. }\end{array}$ & $\begin{array}{l}\text { El alumno es capaz } \\
\text { de analizar diversas } \\
\text { perspectivas de un } \\
\text { mismor hecho } \\
\text { histórico. }\end{array}$ & $\begin{array}{l}\text { El alumno puede } \\
\text { analizar diversos } \\
\text { puntos de vista } \\
\text { para interpretar } \\
\text { el pasado y } \\
\text { comprende que } \\
\text { estos están } \\
\text { condicionados y } \\
\text { son consecuencia } \\
\text { de un contexto } \\
\text { determinado. }\end{array}$ & $\begin{array}{l}\text { El alumno puede analizar } \\
\text { diversos puntos de vista } \\
\text { para interpretar el pasado y } \\
\text { comprende que estos están } \\
\text { condicionados y r son } \\
\text { consecuencia de un } \\
\text { contexto determinado y } \\
\text { además es capaz de realizar } \\
\text { sus propias interpretaciones } \\
\text { si posee suficiente } \\
\text { información sobre el } \\
\text { contexto. }\end{array}$ \\
\hline $\begin{array}{l}\text { Evitar el } \\
\text { presentismo: a } \\
\text { fin de interpretar } \\
\text { las creencias y } \\
\text { comportamientos } \\
\text { de los personajes } \\
\text { históricos del } \\
\text { mejor modo } \\
\text { posible. }\end{array}$ & $\begin{array}{l}\text { El alumno cae en } \\
\text { el presentismo, } \\
\text { puesto r que } \\
\text { extrapola } \\
\text { propios } \\
\text { sentimientos y } \\
\text { actitudes al pasado } \\
\text { y no comprende } \\
\text { que existen } \\
\text { diferencias entre } \\
\text { las creencias y } \\
\text { comportamientos } \\
\text { del pasado y las } \\
\text { actuales. }\end{array}$ & $\begin{array}{lr}\text { El alumno asume } \\
\text { que } & \text { las } \\
\text { mentalidades } & \text { y } \\
\text { actitudes } & \text { son } \\
\text { diferentes en } & \text { el } \\
\text { pasado y } & \text { que } \\
\text { nuestro } & \\
\text { comportamiento } \\
\text { actual no puede } \\
\text { extrapolarse } r \\
\text { pasado pero de } \\
\text { modo inconsciente } \\
\text { cae en r el } \\
\text { presentismo en } \\
\text { algunas ocasiones. }\end{array}$ & $\begin{array}{l}\text { El alumno asume } \\
\text { que re las } \\
\text { mentalidades y } \\
\text { actitudes ron } \\
\text { diferentes en el } \\
\text { pasado y que } \\
\text { nuestro } \\
\text { comportamiento } \\
\text { actual no puede } \\
\text { extrapolarse al } \\
\text { pasado. }\end{array}$ & $\begin{array}{l}\text { El alumno asume que las } \\
\text { mentalidades y actitudes } \\
\text { son diferentes en el pasado, } \\
\text { que nuestro } \\
\text { comportamiento actual no } \\
\text { puede extrapolarse al } \\
\text { pasado y puede mencionar } \\
\text { los factores que provocan } \\
\text { estas diferencias. }\end{array}$ \\
\hline
\end{tabular}

Fuente:Adaptado de Davison (2012a: 123) y Martínez-Rojas (2008: 133) 
La rúbrica es de tipo analítico, orientada a "determinar el grado de comprensión o de manejo o de desempeño de un estudiante en un aspecto particular (...) su nivel de desarrollo" (MartínezRojas, 2008). En este caso, buscamos conocer hasta qué punto los alumnos han conseguido comprender el contexto y los hechos históricos abordados mediante las actividades de empatía histórica. Utilizaremos para ello, tres de las dimensiones de la empatía histórica propuestas por Davison (2012) y en base a estas, estableceremos cuatro niveles progresivos de éxito en su consecución, en función de si se ha logrado una comprensión más o menos profunda (MartínezRojas, 2008).

Asimismo, mediante un breve cuestionario buscaremos conocer la opinión de los alumnos, a fin de determinar si las actividades ha sido motivadora para ellos y creen que puede ayudarles a comprender adecuadamente los contenidos. En la tabla hemos reflejado los porcentajes finales extraídos de los resultados de la encuesta realizada en ambas clases con referencia a las actividades desarrolladas en el aula.

TABLA 5. Cuestionario sobre las actividades de empatía histórica

\begin{tabular}{|c|c|c|}
\hline PREGUNTAS & SI & NO \\
\hline ¿Te gustaría repetir este tipo de actividades en clase? & $82,00 \%$ & $12.00 \%$ \\
\hline ¿Crees que las actividades han sido entretenidas? & $95.00 \%$ & $5.00 \%$ \\
\hline ¿Piensas que estas actividades te ayudan a comprender los contenidos? & $89.00 \%$ & $11,00 \%$ \\
\hline $\begin{array}{l}\text { ¿Crees que recuerdas mejor y puedes aprender más efectivamente analizando y } \\
\text { debatiendo información en clase con los compañeros que siguiendo los métodos de } \\
\text { estudio habituales? }\end{array}$ & $91,00 \%$ & $9.00 \%$ \\
\hline
\end{tabular}

Fuente: Elaboración propia

\section{RESUltados}

A continuación procederemos a exponer los resultados observados tanto en términos de comprensión de los hechos históricos como de motivación académica. Como hemos indicado, nos hemos basado en las dimensiones de la empatía histórica propuestas por Davison (2012a) para evaluar las actividades. Respecto a la primera dimensión: "mentalidad abierta”, hemos observado una notable capacidad de los alumnos para adoptar puntos de vista ajenos en el marco de un contexto determinado durante el desarrollo de la actividad A. En algunos casos los alumnos parten, tanto de los conocimientos que poseen sobre el contexto histórico, como de las habilidades que les proporcionan las actividades de empatía histórica para comprender las mentalidades ajenas. Comparar el presente con el pasado permite al alumno considerar aspectos que no habría considerado o no habría comprendido en el marco de una explicación tradicional de los hechos, como por ejemplo, la confianza que pueda transmitir un determinado líder político y la influencia que este factor pueda tener en su ascenso o impopularidad:

Investigadora: "Hemos observado que los votantes de Hitler y Mussolini no consideraban a estos líderes como políticos profesionales. También en los Estados Unidos comprobamos como los votantes de Donald Trump señalan que él "no es un político como los demás”. En ambos casos sus votantes enuncian esta cualidad como positiva. ¿Por qué?”

Alumno: "Porque pensaban, como nosotros, que los políticos van a engañarnos. Si ves a alguien que no es un político te fías más”.

Investigadora: “¿Y por qué desconfiaban de los políticos en Alemania?” 
Alumna: Porque habían perdido una guerra, habían pasado por una crisis económica...Y Hitler decía que los políticos de la República de Weimar habían traicionado al país, que no habían defendido alemania de la crisis económica y que se habían sometido a las otras potencias que les pedían reparaciones de guerra muy altas.

Alumno: Hitler había luchado en la guerra, había sido un soldado y estaba enfadado por la derrota. Hablaba como un ciudadano más.

No obstante, si bien durante la actividad A los resultados han sido óptimos, en la actividad B los alumnos han presentado mayores dificultades. En este caso han realizado el mismo razonamiento a la inversa. Es decir, en la actividad A los alumnos han utilizado sus experiencias sobre el presente para comprender las motivaciones del pasado. Pero en la actividad B los alumnos han partido de las experiencias del pasado para tratar de dilucidar cómo actuarían ellos mismos en el presente. En este caso, la implicación emocional les ha dificultado enormemente la tarea, y la mayoría de ellos ha considerado que ante una situación de ascenso de un régimen totalitario, se opondría firmemente. Hemos encontrado algunas excepciones, como la que observamos en la autora del siguiente fragmento de una de las cartas: "Sinceramente, no sé lo que haría ya que si lo ves desde fuera (toda esa situación) puedes pensar de una forma distinta a cómo se ve desde dentro. Todos somos muy fáciles de manipular”.

En cuanto a la dimensión "buscar múltiples perspectivas” los alumnos han respondido satisfactoriamente. Las actividades les han ayudado a comprender la multicausalidad y el rechazo de explicaciones simplistas. El visionado de los videos de los votantes durante la actividad A y la película "La Ola” han permitido a los alumnos la observación de los múltiples factores psicológicos que pueden incidir en la adscripción a una determinada ideología según cada individuo, así como las diversas causas políticas y sociales que condicionan cada caso.

Respecto a la dimensión "evitar el presentismo", hemos observado que esta es la que mayor dificultad implica por parte de los alumnos. En las siguientes preguntas, intentamos que los alumnos comprendan que no pueden extrapolar por completo la situación del pasado a la actualidad, que la sociedad no presenta las mismas características pero tampoco es radicalmente diferente:

Investigadora: "Podemos establecer paralelismos entre la situación actual del auge de la extrema derecha y el ascenso de los fascismos ¿Creéis que la sociedad actual podría volver a caer en una dictadura que está igual de desinformada que en el pasado”?”

Alumno: “Ahora ya no podría suceder porque tenemos más conocimientos y otra forma de pensar”.

Investigadora: "Pero realmente...¿¿Han pasado tantos años? ¿Pensáis que somos muy diferentes de las personas que vivieron en la década de los 30 del siglo XX?”

Alumno: Ahora estamos informados, sabemos todo lo que pasa.

Investigadora: “¿Entonces...creéis que estáis bien informados y que por lo tanto podríais evitar una situación así?”

Alumna: Tenemos mucha información, igual demasiada porque hay tantas notícias, en la tele, en internet...que al final no miras nada. O no sabes si lo que dicen es verdad o no.

En términos de motivación, se ha observado una participación activa del alumnado, que ha formulado preguntas, teorías...Las intervenciones durante las clases han sido constantes y los estudiantes han mostrado una notable implicación respecto a las actividades. Asimismo, el cuestionario presentado nos ha revelado que los alumnos encuentran motivadoras este tipo de actividades. Por otro lado, también observamos, en base al cuestionario, la opinión favorable que se deriva de los alumnos respecto a la idoneidad de estas actividades para aprender de un modo más eficiente y significativo.

Como contrapartida, cabe remarcar que si bien las ventajas de aplicar este tipo de actividades en el aula son patentes, el elevado tiempo que se invierte en su preparación limita la aplicación de las mismas. Sería necesaria una mayor comunicación y colaboración docente para diseñar y 
planificar este tipo de actividades, llevando a cabo un intercambio de materiales e ideas que permitiese amortizar el tiempo de preparación de las mismas, así como enriquecer la actividad docente con otros puntos de vista y opiniones.

\section{CONCLUSiOnes}

Del estudio realizado, tanto teórico, como práctico, podemos llegar a una serie de conclusiones. En primer lugar, los ejercicios de empatía histórica permiten evitar el aprendizaje superficial, una acción común entre los estudiantes y que carece de sentido didáctico. Así lo indican los diversos autores que han llevado a cabo este tipo de actividades en el aula (Kohlmeier, 2006; Mootz,2014; Davison, 2012a). Asimismo, Prats remarca la especial idoneidad de las actividades de empatía histórica para lograr un conocimiento adecuado de los hechos históricos, puesto que "No es posible contextualizar los hechos si los alumnos y alumnas no son capaces de demostrar que son conscientes de las motivaciones humanas, con referencia a acontecimientos del pasado" (Prats,2001: 17). Por otro lado, en las actividades que hemos planteado hemos comprobado cómo los alumnos han formulado hipótesis y han analizado en profundidad los hecho presentados, evitando así un aprendizaje superficial. Llevar a cabo una simple memorización de los contenidos únicamente puede permitir al alumnado aprobar la asignatura, sin conseguir ningún tipo de conocimiento que le permita comprender el mundo que le rodea. La Historia no puede ser considerada como una materia que solo requiere memorización. Una disciplina que implica un alto grado de análisis y reflexión y que puede aportar competencias imprescindibles para interpretar el mundo y moverse en él no puede quedar limitada a una escucha pasiva (Canals, 2008).

En segundo lugar, trabajar la empatía histórica en el aula permite estimular la motivación intrínseca de las actividades. Hemos comprobado este aspecto tanto mediante la investigación teórica (Beltrán et al., 1987; Kohlmeier, 2006; Davison, 2012b; Guillén, 2016; Cruz y Pizarro, 2014, Downey, 1995; Kosti, 2015) como a través de la observación del comportamiento del alumnado en el aula y sus propias opiniones sobre las actividades desarrolladas. Debemos considerar que la motivación del alumnado cobra especial importancia en un contexto donde algunos estudiantes pueden no tener ningún tipo de motivación externa para superar con éxito una asignatura. En este contexto, un aprendizaje motivador por sí mismo es, posiblemente, la única forma de conseguir el éxito escolar. La empatía histórica sitúa al alumno como protagonista de su aprendizaje. Los estudiantes tienen un papel activo: investigan sobre personajes históricos, expresan sus reflexiones y opiniones en debates, realizan juegos de rol... Los estudiantes aprenden de una forma dinámica y entretenida. Además, los ejercicios de empatía histórica motivan la curiosidad de los estudiantes, puesto que incluyen interrogantes que estos tienen que resolver, como por ejemplo, en el caso de las actividades que he planteado, averiguar por qué los regímenes fascistas consiguieron el apoyo de la población. Se trata de romper con un aprendizaje rutinario que no sorprende ni plantea ningún reto al alumnado.

Así pues, la empatía histórica, supone un medio para mejorar el aprendizaje y la enseñanza de la Historia. Pero las actividades de empatía histórica necesitan un profesorado que deje a un lado los libros de texto y las lecciones magistrales para llevar a cabo nuevas metodologías en el aula. Un profesorado inquieto, que se cuestione sus propios métodos, reflexione e investigue.

\section{Referencias}

ATRESMEDIA (2017). Ruta Trump. La sexta TV. Recuperado de http://www.lasexta.com/especiales/ruta-trump/ [3 de mayo de 2017].

BBC MUNDO (2017). Los jóvenes de extrema derecha que apoyan a Marine Le Pen en Francia. $B B C$. [Video]. Recuperado de https://www.youtube.com/watch?v=QKTvFAcJalg [1 de mayo de 2017]. 
Beltrán, J., Moraleda, M., Calvo, E., Calleja, F. y Bermejo, V. (1987). Psicología de la Educación. Madrid: Eudema.

Canals, R. (2008). La didáctica de las Ciencias Sociales: Contribución al desarrollo de competencias básicas en la educación obligatoria. En R. M. Ávila, A. Cruz y M. C. Díez, (Eds.), Didáctica de las Ciencias Sociales, Currículo Escolar y Formación del Profesorado. La didáctica de las ciencias sociales en los nuevos planes de estudios (pp. 331-357). Jaén: Universidad de Jaén.

Cruz, P. y Pizarro, F. (2014). Empatia en clase de Historia: Los alumnos serán soldados de la Primera Guerra Mundial. Clio. History and History teaching, 40.

Davison, M. (2012a). "It is Really Hard Being in Their Shoes": Developing Historical Empathy in Secondary School Students (Tesis doctoral). Auckland:University of Auckland.

Davison, M. (2012b). Teaching historical empathy and the 1915 Gallipoli campaign. En M. Hartcourt y M. Sheehan (Eds.), History Matters: Teaching and Learning history in New Zealand schools in the 21st century (pp. 11-31). Wellington: NZCER Pres.

Downey, M. (1995). Doing history in a fifht-grade classroom: perspective taking and historycal thinking. 1995 Annual Meeting Call for Proposals. San Francisco: American Educational Research Association.

Endacott, J. (2010). Reconsidering Affective Engagement in Historical Empathy. Theory \& Research in Social Education, 38(1), 6-47.

Endacott, J. y Brooks, S. (2013). An Updated Theoretical and Practical Model for Promoting Historical Empathy. Social Studies Research and Practice, 8(1), 41-58.

Évole J. y Lara, R. (2017). Hijos de la ira. De Trump a Le Pen I. La Sexta TV. [Video]. Recuperado de https://www.atresplayer.com/ [27 de abril de 2017].

Foster, S. (1999). Using Historical Empathy to Excite Students about the Study of History: Can You Empathize with Neville Chamberlain? The Social Studies, 90(1), 18-24.

Guillén (2016). Un viaje al siglo XVI a partir de los casos de un corsario y un bandolero. Una experiencia de empatía histórica en Secundaria. Clio. History and History teaching, 42.

HISPANTV (2017). Macron y Le Pen se enfrentan en un debate televisivo. [Video]. Recuperado de https://www.youtube.com/watch?v=qB5LSn2xBys [3 de mayo de 2017].

Kohlmeier, J. (2006). “Couldn't she just leave?”: The Relationship Between Consistently Using Class Discussions and the Development of Historical Empathy in a $9^{\text {th }}$ Grade World History Course. Theory and Research in Social Education, 34 (1), 34-57.

Lazarouk, E. (2008). Empathy as a tool for historical understanding: an evaluative approach of the ancient greek primary history curriculum. International Journal of Social Education, 23(1), 27-50.

Martínez-Rojas, J. G. (2008). Las rúbricas en la evaluación escolar: su construcción y uso. Avances en Medición, 6, 129-134.

Mootz, D. G. (2014). Towards a taxonomy: History teachers and History classrooms (Tesis doctoral). Sydney: University of Western Sydney.

Neitzel, S. y Welzer H.(2012) Soldados del Tercer Reich: Testimonios de lucha, muerte y crimen. Barcelona: Crítica.

Pagès, J. (2007). ¿Qué se debería enseñar de historia hoy en la escuela obligatoria?, ¿qué deberían aprender, y cómo, los niños y las niñas y los y las jóvenes del pasado? Escuela de Historia, 6 (1).

Pagès, J. (2008). El lugar de la memoria en la enseñanza de la historia. Íber. Didáctica de las Ciencias Sociales, Geografía e Historia, 55, 43-53.

Pagès, J. y Santisteban A. (2013) Una mirada al pasado y un proyecto de futuro: Investigación e innovación en didáctica de las ciencias sociales. Barcelona: Universitat Autònoma de Barcelona. 
Pericacho, F. J. (2015). Actualidad de la renovación pedagógica en la Comunidad de Madrid: un estudio a través de escuelas emblemáticas. Evolución y experiencias actuales ante los retos socioeducativos de la sociedad del siglo XXI (Tesis doctoral). Madrid: Universidad Complutense de Madrid.

Prats, J. (2001). Enseñar Historia: Notas para una didáctica renovadora. Mérida: Junta de Extremadura.

René, J. (2010). El mito del Duce. Testimonios orales sobre la percepción de Mussolini. En R. Kolter (Coord.), Memoria, Fuentes Orales y Ciencias Sociales: Primeras Jornadas de Historia Reciente del NOA. Tucumán: AHONA.

Sierra Gómez, T. (2013). El aprendizaje activo como mejora de las actitudes los estudiantes hacia el aprendizaje (Trabajo final de máster). Navarra: Universidad de Navarra.

Yilmaz, K. (2007). Historical Empathy and Its Implications for Classroom Practices in Schools. The History Teacher, 40(3), 331-337.

\section{CómO CITAR ESTE ARTÍCULO}

Doñate Campos, O. y Ferrete Sarria, C. (2019). Vivir la Historia: Posibilidades de la empatía histórica para motivar al alumnado y lograr una comprensión efectiva de los hechos históricos. Didáctica de las ciencias experimentales y sociales, 36, 47-60. DOI: 10.7203/DCES.36.12993. 